\title{
Medication induced fetal bladder rupture: a case report
}

\author{
Eldon Palmer ${ }^{1,2}$, Milette Oliveros ${ }^{1}$, Jason Fong ${ }^{3}$, George Graham ${ }^{3 *}$

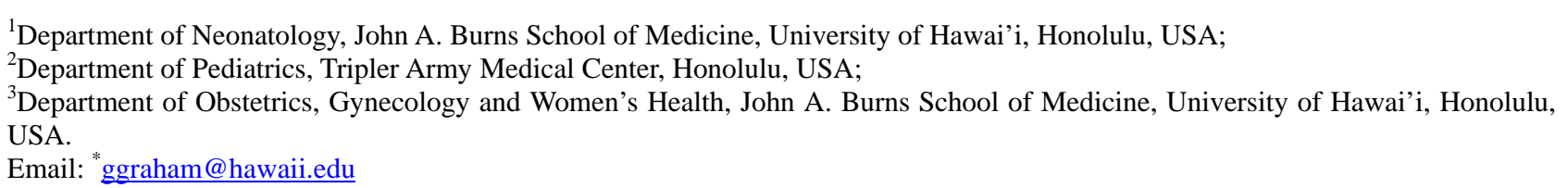

Received 29 March 2011; revised 19 April 2011; accepted 27 April 2011.

\begin{abstract}
BACKGROUND: Intrauterine bladder rupture is a rare complication usually caused by structural bladder outlet obstruction. Some medications are known to cause urinary retention or diuresis in fetuses and preterm infants. CASE: A 31-year-old gravida 6, para 3023 at 29 weeks and 2 days' gestation required intubation, mechanical ventilation, and medical management for severe chest pain and respiratory failure, eventually diagnosed as asthma and pneumonia. An obstetrical ultrasound on hospital day three revealed a markedly dilated fetal bladder. Repeat ultrasound the following day showed a decompressed fetal bladder and significant ascites. A cesarean delivery was performed for a nonreassuring fetal heart rate. Postnatal evaluation by voiding cystourethrogram and cystoscopy revealed bladder rupture without evidence of outlet obstruction. Given the absence of other plausible causes, the rupture was likely due to exposure to maternal medications. CONCLUSION: Transplacental exposure to maternal medications may cause fetal urinary retention and intrauterine bladder rupture. Fetal ultrasound surveillance during treatment with medications known to cause urinary retention may allow for early diagnosis and intervention.
\end{abstract}

Keywords: Bladder Rupture; Maternal Medication; Pharmacology; Fetal Urinary Retention; Fetal Ultrasound

\section{INTRODUCTION}

Megacystis and intrauterine bladder rupture are rare complications usually resulting from structural bladder outlet obstruction. Transplacental exposure to medications during pregnancy has been associated with fetal urinary retention. This is the first case in the literature of fetal urinary retention secondary to maternal medication exposure resulting in intrauterine bladder rupture.

\section{CASE REPORT}

A 31-year-old gravida 6, para 3023 with normal prenatal labs and a normal 22 week ultrasound presented to a local emergency room at 29 2/7 weeks' gestation with symptoms of shortness of breath and severe chest pain. Due to respiratory failure, she was intubated and placed on mechanical ventilation. She was diagnosed with asthma and bacterial pneumonia and transferred to the medical intensive care unit (ICU) of a referral center.

The patient remained on a ventilator and was continued on sedation, pain control, asthma management, and antibiotics. She was given a course of steroids for fetal benefits. An obstetrical ultrasound performed on hospital day three was notable for an enlarged, thin-walled bladder with a mean diameter of $4.8 \mathrm{~cm}$ (Figure 1). There was a trace amount of ascites seen. The amniotic fluid volume appeared normal, with an amniotic fluid index of $20 \mathrm{~cm}$. The renal pelvises measured five mm bilaterally without evidence of dilated ureters. The gender was female. A biophysical profile was reassuring and the remainder of the anatomical survey appeared normal.

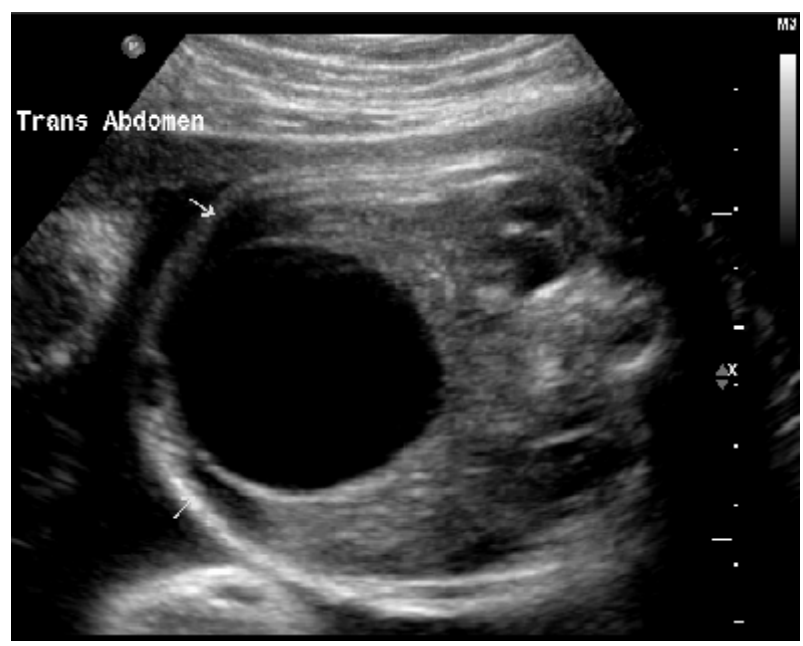

Figure 1. Prenatal ultrasound showing an enlarged fetal bladder. 
On hospital day four, fetal monitoring showed an absence of variability and recurrent late decelerations in the fetal heart rate. A bedside ultrasound revealed a decompressed bladder and significant ascites (images not available).

A cesarean delivery was performed under general anesthesia for nonreassuring fetal status. A female infant weighing $1531 \mathrm{~g}$ with Apgars of 3, 7, and 9 was delivered. On exam the neonate was noted to have a markedly distended abdomen. Respiratory distress persisted beyond the initial resuscitation period and the baby was transferred to a referral neonatal ICU for further evaluation and management. An abdominal ultrasound performed on the first day of life (DOL) showed ascites with mild to moderate left hydronephrosis, left hydroureter, and a decompressed bladder (Figure 2).

Anuria was noted during the first DOL. A peritoneal drain was placed on the second DOL yielding $140 \mathrm{~mL}$ of fluid with an electrolyte profile consistent with urinary ascites. Voiding cystourethrogram (VCUG) showed a small bladder with leakage of contrast into the peritoneal cavity (Figure 3). Cystoscopy revealed a defect in the posterior bladder wall (Figure 4). Importantly, no anatomic bladder outlet obstruction was seen. Foley output improved and peritoneal output eventually stopped on DOL eight. A repeat VCUG on DOL 19 showed no evidence of the previously seen defect. The baby was discharged on DOL 21 with normal bladder function.

The mother did well postoperatively. She was extubated on postoperative day one and discharged on hospital day nine.

\section{DISCUSSION}

This is the first case in the literature of fetal urinary retention and subsequent bladder rupture secondary to maternal medication exposure. Previously described causes of fetal bladder outlet obstruction include urethral atresia, urethral valves, prolapsed ureterocele, megacystis megaureter and megacystis microcolon. Although it is

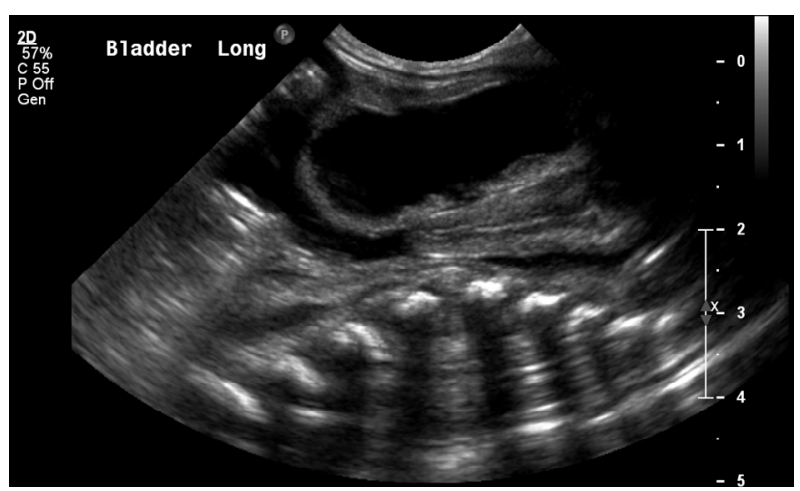

Figure 2. Neonatal ultrasound showing a decompressed bladder and ascites.

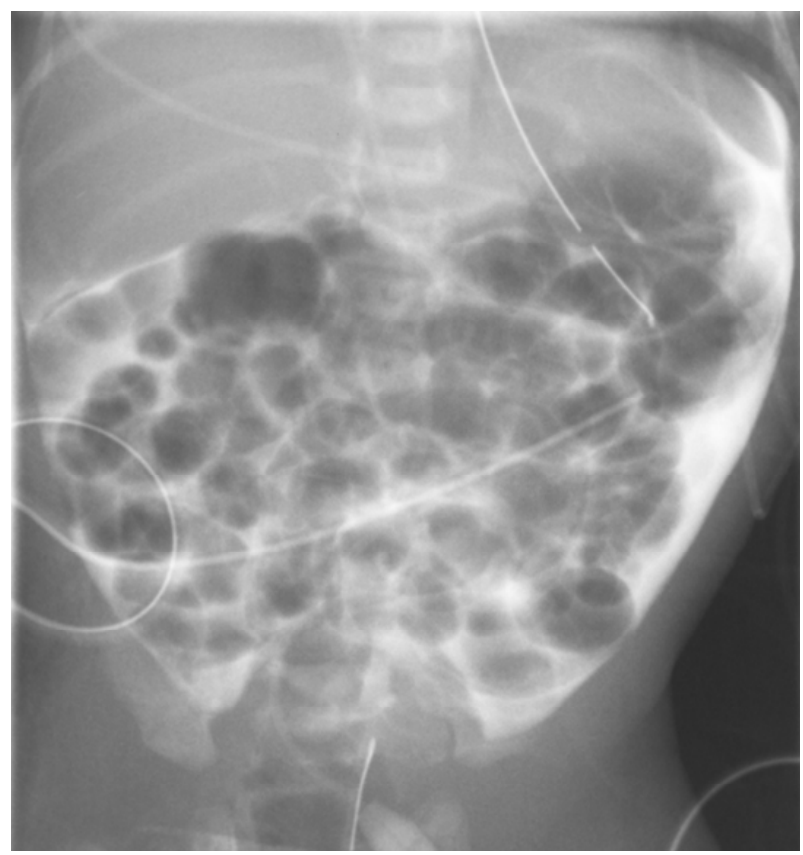

Figure 3. Voiding cystourethrogram showing a contracted bladder with contrast in the peritoneum.

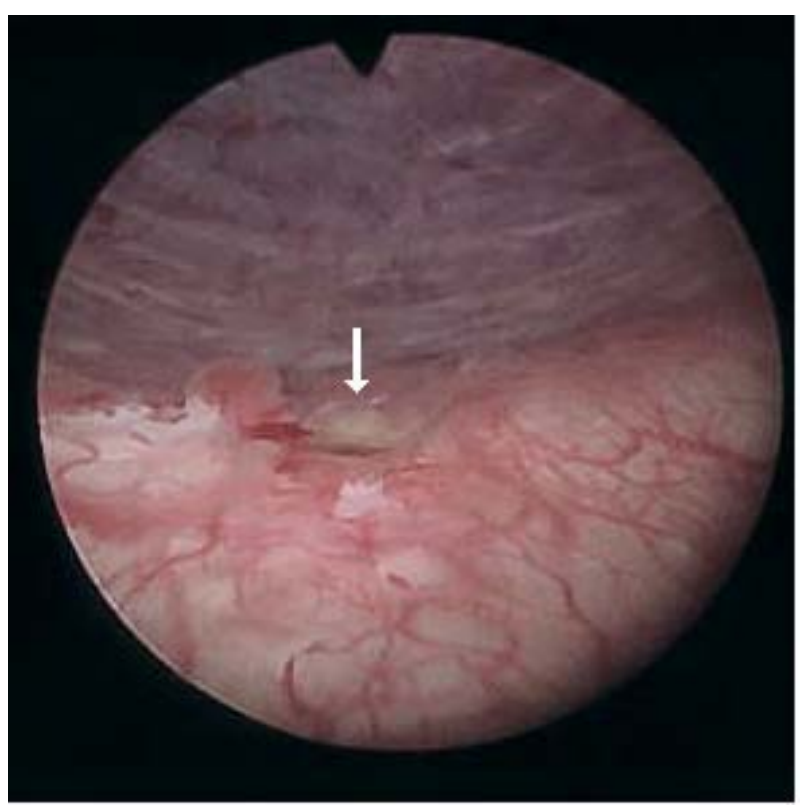

Figure 4. Cystoscopy showing a perforation in the posterior bladder wall.

not possible to prove that fetal urinary retention was due to maternal medication exposure, the postnatal evaluation excluding structural causes of bladder outlet obstruction makes transplacental medication exposure the most likely etiology. This functional obstruction, combined with medication induced diuresis, likely contributed to intrauterine bladder rupture. 
Our diagnosis of acute fetal urinary retention is based on a normal appearing bladder at 22 weeks' gestation, and the subsequent appearance of a thin-walled distended bladder with a normal amniotic fluid volume and normal appearing fetal kidneys. The smooth appearance of the abdomen after drainage of the ascites is also significant, as chronic abdominal distension would likely lead to a prune belly appearance of the abdominal wall.

Transplacental exposure to maternal antipsychotic medications has been proposed as a cause of fetal urinary retention, with subsequent resolution after discontinuation of the medications [1]. We reviewed the maternal medications used in this case for their potential to cause urinary retention and to cross the placenta. Of the medications used, fentanyl, morphine, ipratropium, diltiazem, and aminophylline have been reported to cause urinary retention.

Opioids, specifically morphine and fentanyl, rapidly cross the human placenta with equilibration between the mother and fetus [2]. Opioids decrease the sensation of bladder fullness by blocking parasympathetic nerves and increase the tone of the bladder neck by stimulating sympathetic nerves, both of which can result in urinary retention. Urinary retention requiring bladder drainage has been reported in premature infants within 24 hours of exposure to morphine [3]. Ipratropium bromide is an inhaled anticholinergic medication used for the treatment of asthma and has been associated with urinary retention [4]. Systemic absorption of inhaled ipratropium is minimal and it is not known whether ipratropium crosses the placenta. Calcium channel antagonists, such as diltiazem, reduce contractility of the bladder and can cause urinary retention in adults. Diltiazem crosses the placenta with a similar concentration in the mother and fetus [5]. Aminophylline is a compound of the bronchodilator theophylline and ethylenediamine, and is used for the treatment of asthma. Theophylline has been associated with urinary retention and diuresis. It is thought that theophylline inhibits contractility of the detrusor muscle by increasing cyclic adenine monphos phate [6]. Theophylline crosses the placenta rapidly, with a similar concentration in the mother and fetus [7]. Maternal furosemide has been used to distend the fetal bladder for evaluation of urinary tract anomalies [8].

Maternal rocuronium and propofol were specifically investigated for urinary effects given their infrequent use in pregnancy. Rocuronium is a nondepolarizing, neuromuscular blocking agent that paralyzes skeletal muscle, but not smooth muscle. Paralysis of skeletal muscle results in relaxation of the external urethral sphincter and therefore would not be expected to cause urinary retention. Propofol is a short acting hypnotic agent, which has not been associated with urinary retention.
Due to the nature of the mother's presentation to the outlying hospital, a large number of medications were administered in the work-up and treatment of her underlying medical condition (Table 1). Since the medications used in this case have not previously been associated with fetal bladder rupture, it is likely the dose and combination of medications that contributed to the outcome in this case (Table 2).

Table 1. Maternal medications given prior to first evidence of bladder distension.

\begin{tabular}{ll}
\hline \multicolumn{1}{c}{ Medication } & \multicolumn{1}{c}{ Known urinary effects } \\
\hline Acetaminophen & None \\
Aminophylline & Aminophylline- difficulty voiding, diuresis \\
Azithromycin & rare nephritis, acute renal failure \\
Betamethasone & Early neonatal diuresis \\
Ceftriaxone & rare renal insufficiency \\
Cisatracurium & None \\
Diltiazem & Urinary retention \\
Enoxaparin & None \\
Fentanyl & urinary tract spasm, urinary retention, oliguria \\
Insulin & None \\
Furosemide & Induces diuresis \\
Ipratropium & dysuria, urinary retention \\
Levalbuterol & hematuria \\
Methylprednisolone & None \\
Morphine & urinary tract spasm, urinary retention, oliguria \\
Oseltamivir & None \\
Propofol & Green urine \\
Rocuronium & None \\
\hline &
\end{tabular}

Table 2. Most likely contributors to fetal bladder rupture.

\begin{tabular}{clc}
\hline Medication & \multicolumn{1}{c}{ Urinary Effect } & $\begin{array}{c}\text { Cumulative } \\
\text { Dose }\end{array}$ \\
\hline Fentanyl & $\begin{array}{l}\text { Increase bladder neck tone } \\
\text { Decreases intensity of afferent } \\
\text { distension signal }\end{array}$ & $1750 \mathrm{mcg}$ \\
Furosemide & Induces diuresis & $80 \mathrm{mg}$ \\
Diltiazem & Decreases bladder contractility & $50 \mathrm{mg}$ \\
& $\begin{array}{l}\text { Decrease detrusor contractility } \\
\text { Aminophylline }\end{array}$ & $250 \mathrm{mg}$ \\
& $\begin{array}{l}\text { Induces diuresis. } \\
\text { Increase bladder neck tone } \\
\text { Dorphine sulfate }\end{array}$ & $\begin{array}{l}\text { Decreases intensity of afferent } \\
\text { distension signal }\end{array}$ \\
\hline
\end{tabular}


Periodic ultrasound examinations to evaluate for megacystis could be considered when medications associated with urinary retention are used during pregnancy; especially when multiple medications and high doses are used. If significant bladder distention develops, a change in medication or a vesicocentesis could be considered to decrease the risk of intrauterine bladder rupture.

\section{ACKNOWLEDGEMENTS}

Norman Sato, MD; Ronald Sutherland, MD; Devin Puapong, MD; Janet Burlingame, MD.

\section{REFERENCES}

[1] Okumo, S., Hamada, H., Fujiki, Y., Yasuoka, M., Watanabe, H., Yamada, N., Sohda, S. and Kubo, T. (1999) Transplacental exposure to antipsychotic drugs during pregnancy and megacystis in the fetus. Prenatal Diagnosis, 19, 980-982.

[2] Moises, E.C., de Barros Duarte, L., de Carvalho Cavalli, R., Lanchote, V.L., Duarte, G. and da Cunha, S.P. (2005) Pharmacokinetics and transplacental distribution of fentanyl in epidural anesthesia for normal pregnant women. European Journal of Clinical Pharmacology, 61, 517-
522. doi:10.1007/s00228-005-0967-9

[3] Bengtsson, G.O., Wootton-Gorges, S.L., Poulain, F.R. and Sherman, M.P. (2003) Urinary effects of morphine in preterm infants. Acta Paediatrica, 92, 251-253. doi:10.1111/j.1651-2227.2003.tb00536.x

[4] Pras, E., Stienlauf, S., Pinkhas, J. and Sidi, Y. (1991) Urinary retention associated with ipratropium bromide. DICP, 25, 939-940.

[5] Bregante, M.A., Aramayona, J.J., Fraile, L.J., Garcia, M.A. and Solans, C. (2000) Diltiazem blood pharmacokinetics in the pregnant and nonpregnant rabbit: maternal and foetal tissue levels. Xenobiotica, 30, 831-841. doi:10.1080/00498250050119871

[6] Owens, G.R. and Tannenbaum, R. (1981) Theophyllineinduced urinary retention. Annals of Internal Medicine, 94, 212-213.

[7] Omarini, D., Barzago, M.M., Bortolotti, A., Lucchini, G., Stellari, F., Enfrati, S. and Bonati, M. (1993) Placental transfer of theophylline in an in vitro closed perfusion system of human placenta isolated lobule. European Journal of Drug Metabolism and Pharmacokinetics, 18, 369-374.

[8] Wladimiroff, J.W. (1975) Effect of furosemide on fetal urine production. British Journal of Obstetrics and Gynecology, 82, 221-224. doi:10.1111/j.1471-0528.1975.tb00623.x 